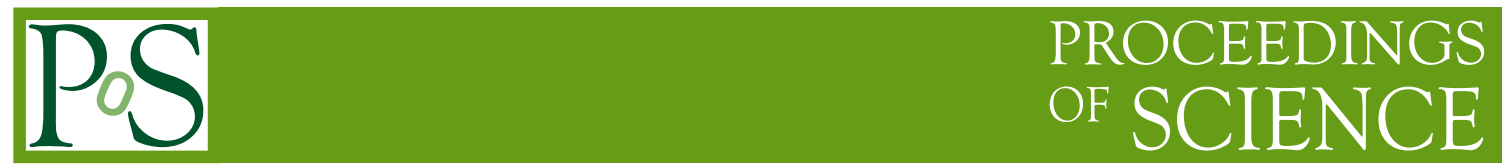

\title{
CVs Astrophysics with LOBSTER and ESA LOFT
}

\author{
René Hudec*t \\ Czech Technical University in Prague, Faculty of Electrical Engineering, Prague, Czech \\ Republic, and Kazan Federal University, Russian Federation \\ E-mail: rene.hudec@gmail.com

\section{Vojtěch Šimon} \\ Czech Technical University in Prague, Faculty of Electrical Engineering, Prague, Czech \\ Republic, and Astronomical Institute, AS CR Ondrejov, Czech Republic \\ E-mail: vojtech.simon@gmail.com
}

The capabilities of the considered space missions LOBSTER and LOFT for investigation of cataclysmic variables (CVs) are discussed.

The Golden Age of Cataclysmic Variables and Related Objects - III, Golden2015

7-12 September 2015

Palermo, Italy

*Speaker.

${ }^{\dagger}$ I acknowledge grant 13-33324S by GACR 


\section{Introduction}

The goal of this paper is to discuss the capabilities of the considered space missions $L O B$ STER and LOFT for investigation of cataclysmic variables (CVs). Both LOBSTER and LOFT can contribute to the study of CVs and related objects. LOBSTER, as a very wide-field soft X-ray monitor, will be able to provide a dense monitoring of the light changes of CVs in X-rays. LOFT can be a promising satellite to provide a sensitive X-ray monitor which will enable to investigate a little studied long-term activity of various types of CVs (especially the magnetic ones) in the X-ray band.

\section{X-ray Sky Surveys}

Most of the past and recent X-ray telescopes used a narrow field of view (FOV) (less than 1 degree diameter). Past X-ray surveys were based mostly on the non-imaging experiments (without a use of optics), hence they were of a limited sensitivity.

Monitors are typically sensitive to the radiation within $2 \mathrm{keV}<E<10 \mathrm{keV}$, hence the soft $\mathrm{X}$-ray emission components often remain unstudied.

ASM/RXTE (All Sky Monitor onboard Rossi X-ray Timing Explorer), was a non-imaging instrument, operating between Jan 1996 and Jan 2012. Several exposures of a given object per day are available in some cases (but in many cases only the daily means are meaningful). The sensitivity of RXTE ASM was good for X-ray binaries with the accreting neutron star or the black hole but only very few CVs $(\sim 10)$ were detectable near the sensitivity limit. Obviously, a new-generation $\mathrm{X}$-ray ASM monitor is needed for CVs.

\section{The importance of monitoring of CVs in X-rays}

$\mathrm{CVs}$ represent the very active objects, with often violent long-term activity in both the optical and X-ray bands (dwarf nova outbursts, high/low state transitions, classical nova explosions), and often with the rapid transitions between the states of activity.

A search for the relation of the optical and X-ray activity is very important, as monitoring of a large number of CVs is necessary to catch them in various states of activity.

Most up to now X-ray observations of CVs are represented by snapshots catching selected CVs in a particular state of activity. In most cases, the transitions between the states are not mapped. There is only poor statistics of phenomena and objects (deeper studies are available only for a few $\mathrm{CVs}$ ). It is obvious that the progress in the future X-ray sky monitoring, as described briefly in this paper, can yield new valuable data to understand the physical processes in CVs in more detail.

The X-ray monitors onboard a given satellite could operate only for a limited time segment: the spectral regions differ for the individual monitors, hence it is difficult and unreliable to combine the data from various monitors. It is necessary to be cautious in assembling the long series of X-ray data.

Most X-ray monitors are sensitive only to the hard or medium X-rays, very few monitors worked at energies $E<1 \mathrm{keV}$. Many pieces of information are thus missing (especially supersoft Xray sources (a special type of CVs may not be detected at all, although they can be very luminous). 


\subsection{Lobster-Eye (LE)}

Lobster-Eye Telescopes represent the novel Wide Field X-ray Telescopes with FOV of 100 sq. deg. and more easily possible (the classical X-ray optics has only 1 deg or less). They represent an analogy with lobster eyes and were designed for astronomy, but laboratory applications are also possible. The Lobster Eye (LE) X-ray optics was originally proposed by Schmidt (1975) and Angel (1979). Since then, numerous test specimens of Lobster Eye telescopes were designed and tested (e.g. Inneman et al. 1999; Hudec et al. 2000, 2003, 2004; Tichý et al. 2009, 2011). The Lobster-Eye (LE) X-ray telescope can be miniaturized for an application in picosatellites. The LE telescopes are based on a real analogy with the lobster eyes.

The LE telescopes can typically serve in two basic operation modes as follows.

- Starrying (pointed) mode - only for the satellite with pointing

- Scanning mode (no satellite pointing and/or stabilization are required)

Parameters of the LE optics are as follows. The energy range from the optical to the energy of $\sim 10 \mathrm{keV}$ in $2 \mathrm{D}$ and from the optical to the energy of $\sim 30 \mathrm{keV}$ in $1 \mathrm{D}$ mode, FOV typical $5 \times 5 \mathrm{deg}$ 1 module, more modules larger FOV, angular resolution $\sim 1$ to $10 \mathrm{arcmin}$ typical, the gain 100 1000. The application areas are as follows. X-ray astronomy in scanning mode, sky monitoring for transients, $\mathrm{X}$-ray astronomy in pointed mode, dense long term monitoring of selected sky area, XRF planetary science, $\mathrm{X}$-ray imaging in the laboratory, $\mathrm{X}$-ray laboratory collimators, $\mathrm{X}$-ray imaging of laboratory plasma, $\mathrm{X}$-ray security screeing and material research, $\mathrm{X}$-ray imaging and low resolution spectroscopy of triggers in atmosphere (balloon experiments), and $\mathrm{X}$-ray imaging and low resolution spectroscopy of polar lights (balloon and rocket experiments).

\subsection{Science objectives of X-ray monitoring}

Wide field X-ray monitors of Lobster Eye type were demonstrated to play an important role in modern astrophysics (e.g. Hudec et al. 2007; Švéda et al. 2004). The most important scientific cases for a monitor directed towards the Galaxy center are briefly summarized below.

- A long-term (months) measurement of the light curves of bright persistent X-ray binaries in the direction toward the center of the Galaxy in the soft X-ray band

- Detection and measurement of the light curves of bright transient events of X-ray binaries in the direction toward the center of the Galaxy in the soft X-ray band

\section{ESA LOFT}

LOFT (the Large Observatory For x-ray Timing) is specifically designed to exploit the diagnostics of very rapid X-ray flux and spectral variability in the compact objects, yielding unprecedented information on strongly curved spacetimes and matter under extreme conditions of density and magnetic field strength (Feroci et al. 2012; Brandt et al. 2013). LOFT is designed to investigate variability from submillisecond quasi-periodic oscillations (QPO) to years long transient outbursts.

LOFT belongs to ESA Medium Class mission (M3 candidate) and was selected in Feb 2011 to complete a 3-year Phase 0/A study. Final down selection on Jan 2014 (5 competed for single 
launch slot) was however negative for LOFT. There is however a chance to be re-submitted in the future ESA calls.

LOFT is an observatory-type mission: there are 2 onboard instruments and a science data center provided by the community. The originally expected launch date was a 2022-2024 timeframe (Soyuz launcher) with 4+1 years mission lifetime and Low Earth orbit $(550 \mathrm{~km}, 2$ ground stations, $7 \mathrm{~Gb}$ science data/orbit).

The LOFT Large Area Detector (LAD) has an effective area $\sim 20$ times larger than any largest predecessor, uniquely combined with a CCD-class energy resolution. LOFT LAD is suitable for observing CVs in a wide X-ray band of $1-40 \mathrm{keV}$, with a very fine time resolution.

The LOFT Wide Field Monitor (WFM) has a 4 steradian field of view at soft X-rays to discover and localize $\mathrm{X}$-ray transients and impulsive events and to monitor spectral state changes, triggering follow-up observations and providing a wealth of science in its own. LOFT WFM is suitable for monitoring of the outbursts and high states of CVs in the energy range of 1-50 keV.

The Czech Technical University in Prague (CTU) is a member of international LOFT consorcium and the considered Czech participation covers several fields, both instrumental, as well as scientific. There is a team at the CTU with an extended expertise in silicon drift detectors and related matters.

\subsection{The LOFT science}

The main LOFT science is a study of the neutron star structure and equation of state of ultradense matter (3+ independent methods): (1) neutron star (NS) mass and radius measurements, and (2) neutron star crust properties. In addition to that, strong gravity and the mass and spin of black holes will be investigated ( 5 independent methods), e.g. quasi-periodic oscillation evolution, and, in the time domain, $\mathrm{nFe}$ line tomography and reverberation studies in bright active galactic nuclei (AGNs) and black hole candidates (BHCs) ((also vs NSs), and relativistic precession. LOFT Observatory Science is expected to provide important observations for virtually all classes of relatively bright sources, including: X-ray bursters, High-mass X-ray binaries, X-ray transients (all classes), CVs, magnetars, gamma-ray bursts (GRBs), nearby galaxies (SMC, LMC, M31...), AGN etc.

In general, CVs are X-ray emitters (e.g. Warner 1995). However, the properties and time evolution of their X-ray spectra strongly depend on the type and the state of activity of a given CV. Although some general relations exist for a given CV type, each $\mathrm{CV}$ is really specific in this regard. Since the proposed band of LOFT is $1-50 \mathrm{keV}$, those CVs with the hardest X-ray spectra will be the suitable targets for observing with this satellite. For example, the intermediate polars usually display the significantly larger values of $\mathrm{k} T$ in comparison with the non-magnetic CVs (Šimon et al. 2006).

As for the onboard instrumentation, LOFT WFM will provide monitoring of CVs flares/high states $1-50 \mathrm{keV}$, while LOFT LAD will provide observations of CVs in hard X-rays (1-40 keV).

Magnetic CVs often have the hard X-ray intensity correlated with the mass accretion rate onto the white dwarf because of the accretion rate onto the magnetic poles of the WD. The dwarf nova outbursts of the intermediate polars can be therefore accompanied by a strong increase of the hard X-ray intensity (e.g. DODra (Szkody et al. 2002). In polars (e.g. Warner 1995), the hard Xray intensity strongly increases in the optical high states. However, the case of the polar AM Her 
shows that the relation of the optical and hard X-ray emission varies for the individual episodes of the high state (Šimon 2011). This emphasizes the necessity to obtain the observations of a larger ensemble of CVs to study the processes and their parameters which influence the behavior of CVs on the long timescales. We therefore expect the satellites like LOFT to be important instruments for investigation of magnetic CVs.

The dwarf nova and intermediate polar GK Per can serve as a very good candidate for the investigations by LOFT (Fig. 1). GK Per is the intermediate polar; accretion from the disk occurs onto the magnetic poles, not via boundary layer. The hard X-ray luminosity thus increases during this dwarf nova outburst (but not in the same way for each such outburst in the same CV). Extinction of radiation is not important in the hard X-ray band like that of BAT: this gives a possibility to constrain the physical model.
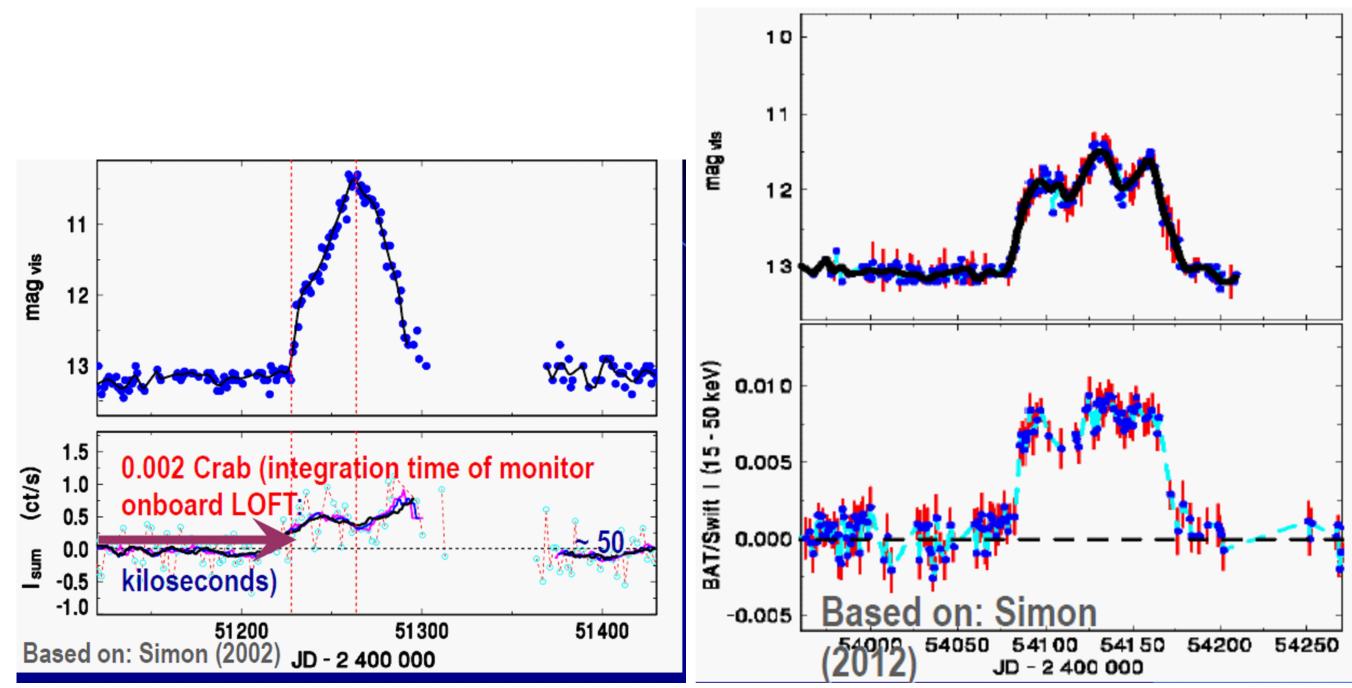

Figure 1: GK Per, one of a very few CVs detected by ASM/RXTE (1.5-12 keV) and BAT/Swift (15-50 keV) with the indicated LOFT WFM sensitivity.

\section{Strategy of observing CVs with LAD/LOFT}

LAD can be used mainly for observing the variations on short timescales (e.g. minutes), also prompt search for the new active states detected by the monitor onboard LOFT is possible.

Folding the data with the orbital period (previously known or found in the LAD data) can pick out even weak signals. The period may be detected only in some spectral regions. In some cases, periodic signal can be observed even in the real time (e.g. an outburst of the intermediate polar). Both the spin modulation of the WD and the superposed longer timescales can be investigated.

\section{Conclusions}

Both LOBSTER as well as LOFT, if approved for space flight, will significantly contribute to the CVs science. 


\section{Acknowledgements}

We acknowledge grant 13-33324S by GACR.

\section{References}

[1] Angel, J. R. P., Astroph. J., 364, 233 (1979)

[2] Brandt, S., et al., 2013, EAS Publications Series, Volume 61, pp.617-623 (2013).

[3] Davidson, K., et al., ApJ, 220, 239 (1978).

[4] Eyer, L. et al., Central European Astrophysical Bulletin, p. 115-126 (2013).

[5] Feroci, M. et al., Experimental Astronomy, Volume 34, Issue 2, pp.415-444 (2012).

[6] Herbig, G. H., et al., ApJ, 141, 617 (1965)

[7] Hudec, L., Algorithms for spectral classification of stars, BSc. Thesis, Charles University, Prague (2007)

[8] Hudec, R., et al., SPIE Proc. 4012, 432 (2000)

[9] Hudec, R., et al., SPIE Proc. 4851, 578 (2003)

[10] Hudec, R., et al., SPIE Proc. 5488, 449 (2004a)

[11] Hudec, R., et al., Nucl. Phys. B Proc. Suppl. 132, 320 (2004b)

[12] Hudec, R., et al., Proc. SPIE 5488, UV and Gamma-Ray Space Telescope Systems, (11 October 2004); doi: 10.1117/12.551915, (2004)

[13] Hudec, R., Šimon, V., Specific object studies for cataclysmic variables and related objects ESA Gaia Reference Code GAIA-C7-TN-AIO-RH-001-1 (2007a).

[14] Hudec, R., Šimon, V., Specific object studies for optical counterparts of high energy sources. ESA Gaia Reference Code GAIA-C7-TN-AIO-RH-002-1 (2007b).

[15] Hudec, R., Pína, L., Inneman, A., Švéda, L., LOBSTER - Astrophysics with Lobster Eye Telescopes, in Exploring the Cosmic Frontier, ESO Astrophysics Symposia European Southern Observatory 2007, pp.73-74, (2007c)

[16] Hudec, R., et al., Acta Polytechnica, IBWS2011 Proceedings, 1(52)(2012)

[17] Hudec, R. et al., Acta Polytechnica, Vol. 53, No. 3, p.30 (2013).

[18] Hudec, R., Šimon, V., Hudec, L., Acta Polytechnica, Vol 53, Supplement, p.798 (2013)

Hudec, R., Astroplate 2014, Proceedings of a conference held in March, 2014 in Prague, Czech Republic. Edited by Linda Miskova and Stanislav Vitek. Published by the Institute of Chemical Technology, Prague, available online at http://astroplate.cz, p.1 (2014)

[19] Inneman, A., et al., Proc. SPIE, 4138, 94 (2000)

[20] Krimm, H. A., et al., ApJS, 209, 14 (2013)

[21] Law, N. M. et al., Publications of the Astronomical Society of the Pacific, Volume 121, issue 886, pp.1395-1408, (2009)

[22] Miceli A. et al., Miceli, A. et al. Astrophys.J. 678, (2008). 
[23] Munari, U., et al., MNRAS, 410, L52 (2011)

[24] Munari, U., et al., MNRAS, 435, 771 (2013)

[25] Pojmanski, G., Acta Astronomica, v.52, pp.397-427, (2002)

[26] Prusti, T., Astronomische Nachrichten, Vol.333, Issue 5-6, p.453, (2012).

[27] Rau, A. et al. Publications of the Astronomical Society of the Pacific, Volume 121, issue 886, pp.1334-1351, (2009)

[28] Šimon, V., Mattei, J. A., A\&AS, 139, 75 (1999)

[29] Šimon, V., A\&A, 360, 627 (2000)

[30] Šimon, V., et al., IAUS, 230, 66 (2006)

[31] Šimon, V., NewA, 16, 405 (2011)

[32] Szkody, P., et al., AJ, 123, 413 (2002)

[33] Schmidt, W. K. H., NucIM, 127, 285 (1975)

[34] Švéda, L., et al., SPIE Proc. 5168, 393 (2004)

[35] Tichý, V., et al., Balt. Astr. 18, 362 (2009)

[36] Tichý, V., et al., Nucl. Instr. Meth. A, A633, S169 (2011)

[37] Tichý, V., et al., SPIE Proc. 8777, 877711 (2013a)

[38] Tichý, V., , SPIE Proc. 8777, 877710 (2013b)

[39] Walker, M., \& Chincarini, G., ApJ, 154, 157 (1968)

[40] Schmidt, M., Ravandoor, K., Kurz, O., Busch, S., Schilling, K., Attitude Determination for the Pico-Satellite UWE-2. In: Space Technology 28. pp.67-74, (2009) 